ISSN: 2697-3391

Vol. 3, N².1, p. 42 - 50, julio, 2020

\title{
Diseño de un programa de la asignatura optativa de Ingeniería de los Alimentos en la carrera licenciatura en nutrición en la Facultad de Ciencias Médicas de Matanzas - Cuba
}

Design of a program of the optional subject of Food Engineering in the degree course in nutrition at the Faculty of Medical Sciences of Matanzas - Cuba.

Odet Knight Romero. ${ }^{1}$ \& Miguel Rodríguez Curbelo. ${ }^{2}$

Recibido: 05-02-2020 / Revisado: 15-03-2020 /Aceptado: 04-04-2020/ Publicado: 05-06-2019

DOI: https://doi.org/10.33262/anatomiadigital.v3i2.1..1260

\begin{abstract}
.
Resumen.

Diet is of great importance as one of the determinants of the health status of individuals and populations, as well as being an essential factor related to the quality of life and survival of the human species. The lack of knowledge about good manufacturing practices, as well as the limited availability of additional technical information, negatively affects food handling and preparation. The objective of this work is to design the program of the optional subject Food Engineering in the Bachelor's degree in Nutrition from the Faculty of Medical Sciences of Matanzas,

La alimentación reviste gran importancia como uno de los determinantes del estado de salud de individuos y poblaciones, además de ser un factor esencial relacionado con la calidad de vida y la supervivencia de la especie humana. La falta de conocimientos sobre las buenas prácticas de manufactura, así como la escasa disponibilidad de información técnica complementaria repercute negativamente en la manipulación y preparación de los alimentos. El objetivo de este trabajo es diseñar el programa de la asignatura optativa Ingeniería de los Alimentos en la carrera Licenciatura en

\footnotetext{
${ }^{1}$ Universidad de Ciencias Médicas de Matanzas, Miembro Titular de la Sociedad Cubana de Enfermería, Matanzas Cuba, odet.mtz@infomed.sld.cu iD https://orcid.org/0000-0002-1240-338x

${ }^{2}$ Universidad de Ciencias Médicas de Matanzas. Miembro Titular de la Sociedad Cubana de Enfermería, Matanzas Cuba miguelr.mtz@infomed.sld.cu iD https://orcid.org/0000-0002-7412-7843
} 
belonging to the Medical University of Matanzas. For which the scientific method supported in the documentary analysis of different programs of the Food Engineering career developed by different authors and normative methodological documents was used. The designed program has a duration of 40 hours distributed in five topics, with conferences, practical classes, seminars and included visits to centers of elaboration, food processing, development of papers and evaluative practical work. The design of the program of the optional subject Food Engineering in the Bachelor's degree in Nutrition allows a greater integration in the design and control of food processing and guarantees a comprehensive vision for the graduate of this specialty.

Key Words: Food, nutrition degree, food engineering.
Nutrición de la Facultad de Ciencias Médicas de Matanzas, perteneciente a la Universidad Médica de Matanzas. Para lo cual se utilizó el método científico sustentado en la realización del análisis documental de distintos programas de la carrera Ingeniería de los Alimentos desarrollados por diferentes autores y documentos metodológicos normativos. El programa diseñado tiene una duración de 40 horas distribuidas en cinco temas, con conferencias, clases prácticas, seminarios e incluyó visitas a centros de elaboración, procesamiento de alimentos, desarrollo de ponencias y trabajos prácticos evaluativos. El diseño del programa de la asignatura optativa Ingeniería de los Alimentos en la carrera Licenciatura en Nutrición permite una mayor integración en el diseño y control del procesamiento de alimentos y garantiza una visión integral para el egresado de esta especialidad.

Palabras Clave: Alimentación, licenciatura en nutrición, ingeniería de los alimentos.

\section{Introducción}

La alimentación reviste gran importancia como uno de los determinantes del estado de salud de individuos y poblaciones además de ser un factor esencial relacionado con la calidad de vida y la supervivencia de la especie humana. Dentro del fenómeno alimentario se entrelazan íntimamente tanto la nutrición como la higiene de los alimentos. La salud y la vida de las personas dependen en gran medida de la calidad nutricional de los alimentos que consumen diariamente, que a su vez depende de la inocuidad higiénico- sanitaria a que estos son sometidos en toda la cadena productiva (Díaz, Cardona, 2015; Fernández et al, 2018; López et al, 2018).

El término industria alimenticia abarca un conjunto de actividades industriales dirigidas al tratamiento, la transformación, la preparación, la conservación y el envasado de productos alimenticios. Las materias primas utilizadas son de origen vegetal o animal y se producen en explotaciones agrarias, ganaderas y pesqueras(Trujillo, Duarte, 2017).

La alimentación hospitalaria se transforma en un proceso complejo, donde la nutrición y dietética, la bromatología, el control de los alimentos, las tecnologías de los alimentos se unen para dar al 
paciente una alimentación, inocua y nutritiva, adecuada a su patología, que contribuya a disminuir la morbimortalidad y proporcionar un mayor estado de bienestar, ya que es parte importante del tratamiento y de la atención a los pacientes (Caracuel, 2014;Betancourt et al, 2016; Fernández et al, 2018; Rivas, 2018).

Los hospitales incorporan servicios de cocina de tamaño y volumen de operaciones nada desdeñables, y en ellos se elaboran y sirven diariamente grandes cantidades de alimentos. Por otro lado, en muchos de los pacientes hospitalizados concurren la vulnerabilidad biológica, la inmunodepresión y la desnutrición (Díaz, Cardona, 2015).

La falta de conocimientos sobre las buenas prácticas de manufactura, así como la escasa disponibilidad de información técnica complementaria repercute negativamente en la manipulación y preparación de los alimentos (Caracuel, 2014).

Una alternativa de solución a estos problemas es el desarrollo de alimentos y estrategias alimenticias tendientes a mejorar el estado de salud de la población mediante la aplicación de la Ingeniería en Alimentos. Esta disciplina permite estudiar la naturaleza de los alimentos, las causas de su deterioro y los principios para su procesamiento y transformación (Zúñiga, Caro, 2017).

El perfeccionamiento de los planes de estudios, la incorporación de nuevos programas en la educación posibilita que el perfil universitario de formación del nutricionista pueda enfrentar nuevos y superiores retos en aras de preservar el estado nutricional de sujetos y colectividades en el país(Plasencia et al,2015;Carbajala, Sierra, López, y Ruperto,2020).

El Licenciado(a) en Nutrición debe velar por el cumplimiento de las políticas de salud vigentes. Participar en la formulación, ejecución y evaluación de Programas de Salud en su área. Brindar educación nutricional individualizada al paciente adulto y pediátrico de los diferentes servicios del Hospital, así como a los usuarios de consulta externa realizando diferentes tipos de encuestas de consumo de alimentos y la evaluación antropométrica.

Realizar actividades de promoción, diagnóstico y recuperación de la salud y estado nutricional óptimo (Hurtado, 2015). El objetivo de este trabajo es diseñar el programa de la asignatura optativa Ingeniería de los Alimentos en la carrera Licenciatura en Nutrición.

\section{Materiales y métodos}

La asignatura de Introducción a la Ingeniería de Alimentos se inserta en el ciclo general de formación del Ingeniero de Alimentos y contribuye como elemento introductorio para el futuro profesional en términos de un abordaje inicial al área de Ciencia, Tecnología e Ingeniería de los Alimentos (Eguez, 2007). 
La asignatura optativa Ingeniería de los Alimentos del Plan " $D$ " de la carrera Licenciatura en Nutrición fue diseñada y redactada por un equipo de profesionales de la Facultad de Ciencias Médicas de Matanzas perteneciente a la Universidad de Ciencias Médicas de Matanzas.

Se utilizó el método científico sustentado en la realización del análisis documental de diferentes programas de la carrera Ingeniería de los Alimentos desarrollados por diferentes autores y documentos metodológicos normativos (Díaz, Cardona, 2015; Ministerio de Educación Superior(MES), 2018).

\section{Resultado y discusión}

La duración del programa es de 40 horas distribuidas en cinco temas: conferencias, clases prácticas, seminarios, visitas a centros de elaboración, procesamiento de alimentos, desarrollo de ponencias y trabajos prácticos evaluativos.

Tabla 1. Temáticas del Programa de Ingeniería de los Alimentos. Matanzas 2019. Los cinco temas se desglosan de la siguiente manera:

Tema 1: Ciencia de los alimentos. Definición de alimentos. Clasificación de alimentos. Composición de los alimentos

Objetivo: Comprender los conceptos fundamentales de los alimentos y su clasificación en función a sus cualidades nutricionales

Tema 2: Microbiología de los alimentos. Microorganismos, definición y clasificación. Bacterias. Reproducción. Tipos de interés para alimentos. Levaduras. Condiciones para el desarrollo. Tipos de Interés. Mohos. Condiciones para el desarrollo. Tipos de interés. Virus. Condiciones

Objetivo: Identificar los microorganismos causantes de alteración en la calidad de los alimentos

Tema 3: Intoxicación de origen alimentario. Envenenamiento producido por ingestión de sustancias tóxicas. Enfermedades ocasionadas por microorganismos que usan el alimento como medio de transmisión. Enfermedades ocasionadas por microorganismos que usan el alimento como medio de crecimiento

Objetivo: Identificar los tipos de intoxicación alimentarias y su origen

Tema 4: Métodos de conservación de alimentos. Principios de conservación. Altas temperaturas. Bajas temperaturas. Agentes químicos

Objetivo: Explicar los tipos de conservación de alimentarias a partir de las características de cada uno de ellos

Tema 5: Limpieza y desinfección en la industria alimenticia. Tipos de limpieza. Fases de limpieza. Limpieza y desinfección. Sistemas de limpieza

Objetivo: $\quad$ Explicar los tipos de limpieza y desinfección existentes 
Tabla 1. Temáticas del Programa de Ingeniería de los Alimentos. Matanzas 2019.

\begin{tabular}{|c|c|c|c|c|c|c|c|}
\hline \multirow[t]{2}{*}{ No. } & \multirow[t]{2}{*}{ TEMATICAS } & \multicolumn{5}{|c|}{$\begin{array}{c}\text { HORAS POR FORMAS } \\
\text { ORGANIZATIVAS DE LA } \\
\text { ENSENAZA }\end{array}$} & \multirow[t]{2}{*}{ TOTAL } \\
\hline & & $\mathbf{C}$ & $\mathbf{C P}$ & $\mathbf{S}$ & ET & TI & \\
\hline $\mathbf{I}$ & Ciencia de los alimentos & 2 & 2 & - & - & 4 & 4 \\
\hline II & $\begin{array}{l}\text { Microbiología de los } \\
\text { alimentos }\end{array}$ & 4 & 4 & 2 & 2 & 10 & 12 \\
\hline III & $\begin{array}{l}\text { Intoxicación de origen } \\
\text { alimentario. }\end{array}$ & 4 & 2 & 2 & - & 6 & 8 \\
\hline IV & $\begin{array}{l}\text { Métodos de conservación de } \\
\text { alimentos }\end{array}$ & 2 & 2 & 2 & 2 & 10 & 8 \\
\hline $\mathbf{V}$ & $\begin{array}{l}\text { Limpieza y desinfección de } \\
\text { los alimentos en la industria } \\
\text { alimenticia }\end{array}$ & 2 & 2 & 2 & 2 & 10 & 8 \\
\hline & TOTAL & 14 & 12 & 8 & 6 & 40 & 40 \\
\hline
\end{tabular}

Fuente: Elaboración propia

Las temáticas abordadas en el programa coinciden con las desarrolladas por diferentes centros de educación superior. Eguiés Montes, (2007) en el programa analítico de la asignatura "Introducción a la Ingeniería de Alimentos" expone nueve temáticas a tratar, de las cuales cinco coinciden con las propuestas en el programa de la asignatura optativa.

El programa cuenta con un total de 32 horas en clases distribuidas entre conferencias, clase práctica y educación en trabajo similar al propuesto la Universidad del Amazona, (2010), además 40 horas de trabajo independiente valor superior al expuesto por la mencionada institución. El programa elaborado aborda temas relacionados con el proceso de vigilancia de la inocuidad, proyectos orientados a la prevención o la solución de problemas de salud relacionados con la ingesta de alimentos, o la oferta de productos que satisfacen necesidades que tienen que ver con la educación alimentaria y nutricionaltal y como refiere Olea; et al, (2019)

El programa elaborado se relaciona con otras asignaturas de la especialidad como: higiene de los alimentos, evaluación de riesgos y técnicas de elaboración de alimentos; buscando la integralidad aspecto abordado por Prapasuwannakul y Bussaban, (2019) en el desarrollo de la ciencia y tecnología de los alimentos.

La asignatura Ingeniería de los alimentos garantiza un mayor nivel de integración del conocimiento en el diseño y control del procesamiento de alimentos conforme al programa de Ingeniería de 
Alimentos propuesto por Facultad de Ingeniería, (2018). No obstante, hay que recordar como plantea Coronel; et al, (2019) que la especialidad de nutrición se inicia en los hospitales ya que muchos programas se elaboraron asociados a facultades o escuelas de medicina.

\section{Conclusiones}

- El programa de la asignatura optativa Ingeniería de los Alimentos se diseñó según la metodología comprendida en la Resolución No.2-2018. Reglamento de Trabajo Docente y Metodológico de la Educación Superior (MES, 2018).

- Los temas se abordan desde una perspectiva ingenieril, pero teniendo en cuenta que están dirigidos a alumnos de muy diferente formación. Permitiendo que adquieran conocimientos sobre normas sanitarias, desarrollo de productos, diseño de procesos, gestión y aseguramiento de la calidad e inocuidad, gestión de la cadena de suministro, investigación, así como desarrollo de proyectos.

- El Licenciado en Nutrición podrá asegurarle a pacientes y familiares que los alimentos procesados que adquieren son inocuos, libres de componentes o agentes que puedan causarles algún tipo de patología o alteraciones en la salud, garantizando la seguridad alimentaria ademáslo convierte en un experto con una visión integral como egresado de esta especialidad .

\section{Referencias bibliográficas}

Caracuel García, Á. M. (2014). Normalización en alimentación hospitalaria y otros establecimientos de restauración social. In S. A. Campofrío FoodGroup (Ed.), (pp. 173). Recuperado de:

Carbajala, Á., Sierra, J. L., López-Lora, L., y Ruperto, M. (2020). Proceso de Atención Nutricional: Elementos para su implementación y uso por los profesionales de la Nutrición y la Dietética Revista Española de Nutrición Humana y Dietética, 24(2), 1-32. https://www.renhyd.org/index.php/renhyd/article/view/961doi: 10.14306/renhyd.24.2.961

Coronel Núñez, S., Pineda Sales, I., Díaz García, R., y Reyes Jorge, J. (2019). Motivos de los estudiantes de la zona centro de méxico para elegir la licenciatura en nutrición. Revista Salud Pública y Nutrición, 18(1), 1-8. : https://doi.org/10.29105/respyn18.1-1

Díaz Lorenzo, T., Cardona Gálvez, M. (2015). Las buenas prácticas de manipulación de alimentos en el hospital Revista Cubana de Alimentación y Nutrición, 25(1), 162-183. Recuperado de: https://www.medigraphic.com > pdfs > revcubalnut > can-2015

Eguez Montes, H. B. (2007). Programa analítico de la asignatura "Introducción a la Ingeniería de Alimentos" (IAL 200) In (pp. 6). Santa Cruz de la Sierra, Bolivia: Universidad Autónoma "Gabriel Rene Moreno“. Recuperado de: https://www.biblio.fcet.uagrm.edu.bo > Carrera > Alimentos $>$ anterior $>$ recursos $>$ INT... 
Facultad de Ingeniería. (2018). Programa de Ingeniería de Alimentos. In (pp. 68). Bogotá: Fundación Universitaria Agraria de Colombia. Recuperado de: https://www.uniagraria.edu.co/wp-content/uploads/2018/06/pep-alimentos-2018.pdf

Fernández, N., Cabral de Bejarano, S., Estigarribia, G., Ortiz, A., y Ríos, P. (2018). Condiciones higiénico-sanitarias basadas en las cinco claves de la OMS de los servicios de alimentación de hospitales del departamento de Caaguazú, Paraguay. Mem. Inst. Investig. Cienc. Salud, 16(2), 21-31. Recuperado de: https://www.revistascientificas.una.py > index.php > RIIC > article > download

Hurtado Escobar, A. S. (2015). Desarrollo de un plan de sanitación y manipulación de alimentos en el área de producción del hospital del IESS de Ibarra. (Administrador gastronómico), Universidad Tecnológica Equinoccial, Quito, Ecuador. Recuperado de: https://www.repositorio.ute.edu.ec > bitstream

López Palomino, M., Amable Ambrós, Zoraida, Garrido Amable, O., y Martínez Fuentes, A J (2018). Estado de los conocimientos y comportamientos sobre alimentación de las personas atendidas en un consultorio del programa del médico de familia. Revista Cubana de Alimentación y Nutrición, 28(2), 463-471. Recuperado de: https://www.medigraphic.com/pdfs/revcubalnut/can-2018/can182n.pdf.

Ministerio de Educación Superior (MES). (2018). Resolución No.2-2018. Reglamento de Trabajo Docente y Metodológico de la Educación Superior. In (pp. 67). Cuba: MES. Recuperado de: https://www.mes.gob.cu/es/resoluciones

Olea Rodríguez, M. A., Venegas Ruiz, B., Martínez Cárdenas, C., Rojas Romero, A. E., \& Michel Valdivia, E. (2019). Implementación del Aprendizaje Basado en Proyectos como Metodología de la Enseñanza en asignaturas de Ingeniería de Alimentos y Biotecnología en el CUCEI/UDG Avances de Investigación en Inocuidad de alimentos, 2. Recuperado de: https://www.e-gnosis.udg.mx/index.php/trabajosinocuidad

Plasencia Concepción, D., Martín González, I., Prado Legrá, E. (2015). Sobre la evolución histórica de la Licenciatura en Nutrición en Cuba. Revista Cubana de Alimentación y Nutrición, 25(2), 411-419. Recuperado de: https://www.medigraphic.com/pdfs/revcubalnut/can-2015/can152o.pdf

Prapasuwannakul, N., y Bussaban, K. (2019). The Relationship between the Academic Performances in Required Major Courses and the Core Courses Achievement of Food Science and Technology Students.International Journal of Information and Education Technology, 9(7), 511-514. doi: 10.18178/ijiet.2019.9.7.1256

Rivas, V. (2018). Sobre los servicios hospitalarios de alimentación y nutrición Revista Cubana de Alimentación y Nutrición, 29(1), 29-31. Recuperado de: https://www.revalnutricion.sld.cu/index.php/rcan/article/view/730

Trujillo Trujillo, Y., Duarte Plata, N. J. (2017). Diseño de estrategia sobre manipulación de alimentos en la asociación pan de vida cer. (Ingeniero Industrial), Universidad Católica De Colombia, Bogotá. Recuperado de: https://repository.ucatolica.edu.co 
Universidad de Amazona. (2010). Programa de curso Introducción a la Ingeniería In (pp. 5): Universidad del Amazona. Recuperado de: https://chaira.udla.edu.co > acreditacion > Pagina > Resources > PDF

Zúñiga Carrasco, I. R., Caro Lozano, J. (2017). Enfermedades transmitidas por los alimentos: una mirada puntual para el personal de salud. Enfermedades Infecciosas y Microbiología, 37(3), 95-104. Recuperado de: https://www.medigraphic.com/cgibin/new/resumen.cgi?IDARTICULO=86155\&id2= 


\section{PARA CITAR EL ARTÍCULO INDEXADO.}

Knight Romero, O., \& Rodríguez Curbelo, M. (2020). Diseño de un programa de la asignatura optativa de Ingeniería de los Alimentos en la carrera licenciatura en nutrición en la Facultad de Ciencias Médicas de Matanzas - Cuba. Anatomía Digital,3(2.1.), 42-50. https://doi.org/10.33262/anatomiadigital.v3i2.1.1260

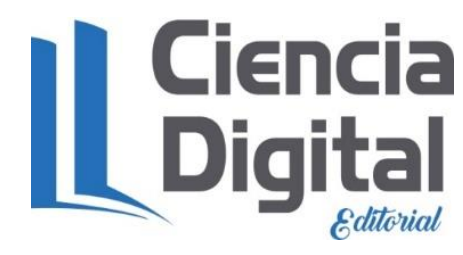

El artículo que se publica es de exclusiva responsabilidad de los autores y no necesariamente reflejan el pensamiento de la Revista Anatomía Digital.

El artículo queda en propiedad de la revista y, por tanto, su publicación parcial y/o total en otro medio tiene que ser autorizado por el director de la Revista Anatomía Digital.
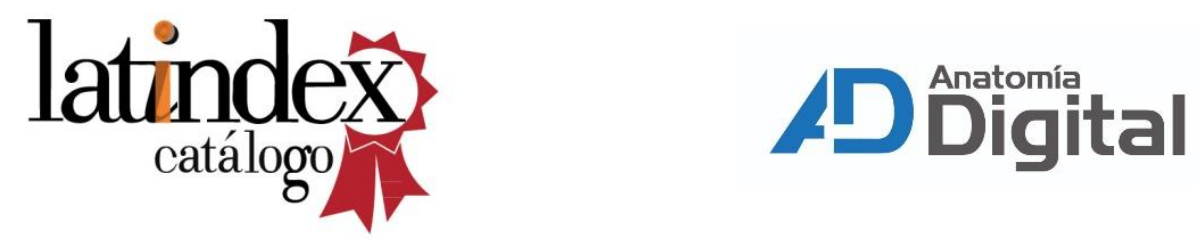